\title{
Confocal Raman AFM imaging of Pharmaceutical Samples
}

\author{
U. Schmidt, A. Jauss, T. Dieing, F. Vargas, and O. Hollricher \\ WITec GmbH, Hoervelsingerweg 6, 89081 Ulm, Germany
}

A thorough knowledge of structural and chemical properties is essential for the development of new medical devices, drug delivery solutions and biomedical applications. These developments require the most detailed information possible concerning the structure and composition of a device surface to better understand and predict how the device will interact with the human body. As these surfaces consist mainly of thin, transparent coatings, surface characterization tools which reveal what is hidden in optical images are required.

Confocal Raman Microscopy combines high resolution microscopy with the chemical sensitivity of Raman spectroscopy, thus allowing nondestructive imaging of chemical properties without special sample preparation. Due to the confocal principle, depth information on the coatings can be easily obtained. Not only can thickness and uniformity measurements be performed, but the degree of mixing or segregation of the ingredients within the coatings can also be determined [1-3].

In addition to the distribution of various chemical species, the presence of drug polymorphs can also be identified using Raman spectroscopy. Even though the Raman spectra of amorphous and crystalline drugs differ only slightly, they can be clearly distinguished with an extremely sensitive confocal Raman setup [4].

In tablets the active pharmaceutical ingredient is distributed in a filler matrix with one or several layers of coatings for easy swallowing and controlled drug release. Such coatings are difficult to be inspected with classical microscopy due to too low or no contrast between individual layers. Fig. 1 a shows the optical image of a coated pellet, where the presence of two coatings is not distinguishable. A 2D-array of 170x150 complete Raman spectra were recorded from a sample area of 170x150 $\mu \mathrm{m}^{2}$ (red marked area). From the 25500 recorded Raman spectra three different spectra were distinguished (Fig. 1 b). The distribution of the three materials is shown in Fig. 1 c-e. These three images were overlayed into a color coded image (Fig. $1 \mathrm{f}$ ). The color of the spectrum matches the color of the Raman image. Using the Raman imaging technique the different coating layers of the pellet (red marked in Fig. 1 a) are identified.

Particle size and their distribution within emulsions can be studied using the confocal Raman imaging technique. For such studies the emulsion is placed between two glass cover slips and imaged with either an oil immersion objective or a cover glass corrected air objective. Fig. 2 a shows characteristic Raman spectra from a four component commercial body lotion. The distribution of the ingredients is presented in Fig. 2 b.

Confocal Raman microscopy can also be used for a label free detection of subcellular organells based on their biochemical composition. This opens the door for noninvasive, in vitro studies of cell biological aspects such as dynamics of mitochondrial movement, drug uptake, and others [5].

Aim of this contribution is to outline the role of confocal Raman microscopy in pharmaceutical studies.

References

[1] K. M. Balss, G. Llanos, G. Papandreou, and C. A. Maryanoff, J. Biomed. Mater. Res. 85 A, (2008) 258.

[2] A. Belu, C. Mahoney, K. Wormuth, J. Controlled Release 126 (2008) 111. 
[3] K. M. Balss, F. H. Long, V. Veselov, A. Orana, E. Akerman-Revis, G. Papandreou, and C. A. Maryanoff, Anal. Chem. 80 (2008) 4853.

[4] K. Wormuth, WITec Customer Report (www.witec.de)

[5] C. Matthäus, T. Chernenko, J. Newmark, C. M. Warner, and M. Diem, Biophys. Journal 93, (2007) 668.
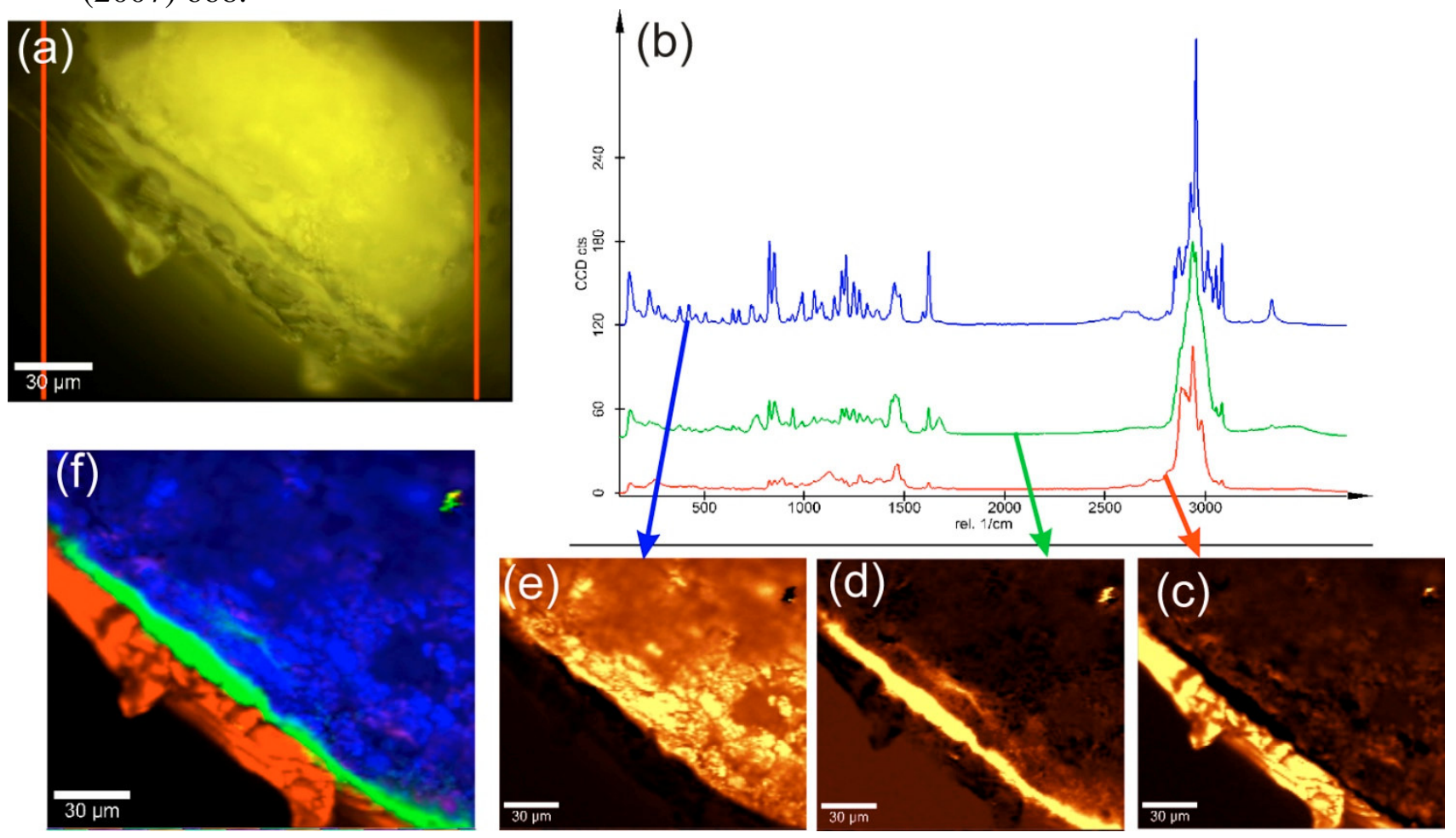

FIG. 1. Video image of a coated pharmaceutical pellet (a), Raman spectra identified in the imaged area (b), distribution of individual chemical species (c-d), and color coded Raman image of the coated pellet (e).

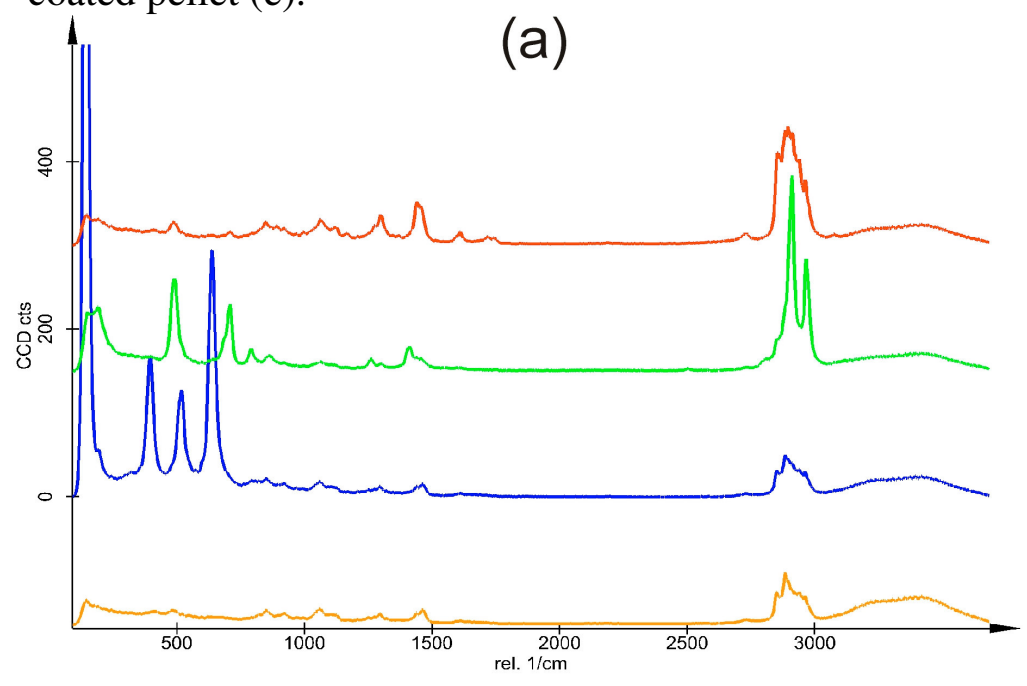

(b)

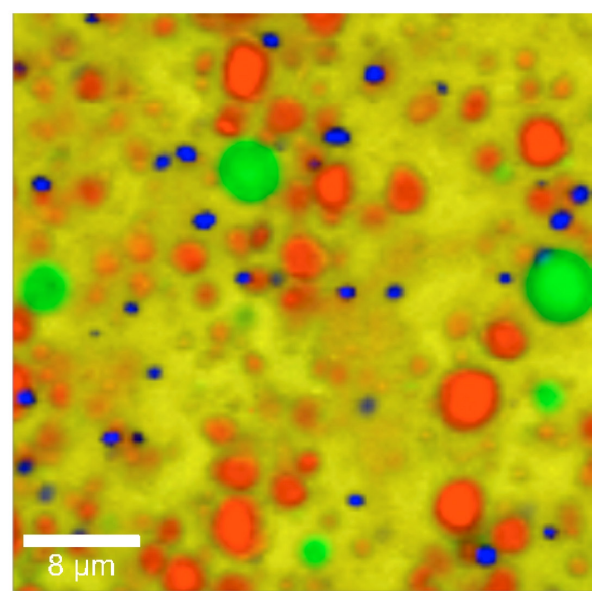

FIG. 2. Raman imaging of a commercial body lotion. Characteristic Raman spectra of the lotion ingredients (a) and color coded Raman image of the lotion revealing the distribution of the lotion ingredients (b). 